\title{
ENERGY SPREAD FROM RF AMPLITUDE AND PHASE ERRORS
}

\author{
L. Merminga and G. A. Krafft, \\ Thomas Jefferson National Accelerator Facility, Newport News, Virginia, USA
}

\begin{abstract}
The energy spread in the beam due to rf amplitude and phase errors is calculated, for multiple passes and off-crest operation. The effects of the slow phase errors and the effects of feedback systems are included in the calculation. If an rms energy spread requirement is to be met, a final formula gives the trade-off between slow and fast phase errors, and amplitude errors. Phase and amplitude stability requirements are derived for the CEBAF IR DEMO FEL.
\end{abstract}

\section{INTRODUCTION}

- Ideally the rf system is timed so the bunches arrive synchronized with the rf; the bunch centroid (in phase) could coincide with the rf crest (on crest operation), or be at a phase $\Phi_{i}$ with respect the crest of the rf wave (off-crest operation).

The beam emerging from the ideal accelerator has a finite energy spread because of the finite energy spread at injection and because of the finite bunch length. In a real accelerator where the effects of the longitudinal wake are negligible, there are contributions to the energy spread from voltage errors and (at least) two types of phase errors. These two types of phase errors are distinguished mainly by the time scale over which the error changes. In this paper, any phase error at frequencies beyond the vernier system bandwidth is called a fast phase error. An example of a fast phase error is a phase difference between the voltage in a cavity and the master oscillator phase line. The second type of phase errors are the ones that change slowly enough that the vernier system is able to respond. In this paper such errors are called slow errors. Thermal expansion phase line errors are examples of slow phase errors.

In the following the rms energy spread is evaluated using two suppositions about the cavity phase and voltage errors. An optimistic result is obtained by assuming that the phase errors are not correlated with the voltage errors and that the different cavities are independent. At the other extreme, we pessimistically assume that the errors are totally correlated. The calculation is carried out for multiple passes and offcrest operation. In the limit of single pass, on crest operation we recover earlier results [1]. We conclude by applying the derived expressions to calculate if phase and amplitude tolerances for the specified energy spread, for the CEBAF IR DEMO FEL.

\section{ENERGY SPREAD CALCULATION}

Let $N$ be the number of cavities in the accelerator. The energy of an electron after $k$ passes through the accelerator is

$T=E+\sum_{n=1}^{N} E_{n}\left(1+A_{n}\right) \sum_{i=1}^{k} \cos \left(\Phi_{i}+\phi_{n}-\Phi_{0}+\Phi+\delta_{n}\right)$

where $E_{n}$ is the voltage set point of the $n$-th cavity, $A_{n}$ is the relative voltage error at the $n$-th cavity, $E$ is the injection energy, $\Phi_{i}$ is the phase set point of the electron bunch with respect to the crest of the rf wave at the $i$-th cavity, $\phi_{n}$ is the slow phase error from the master oscillator to the $n$ th cavity, $\Phi_{0}$ is the injector phase offset, $\Phi$ is the injection phase of the particle (due to the finite bunch length), and $\delta_{n}$ is the fast phase error to the $n$-th cavity. The goal is to compute the energy spread as a function of the slow phase errors $\phi_{1}, \ldots, \phi_{N}$.

When the cavity phase and voltage errors are uncorrelated the probability density function is a product of the independent probability densities

$$
F\left(E, \Phi, \delta_{1}, A_{1}, \ldots \delta_{N}, A_{N}\right)=f(E, \Phi) \prod_{n=1}^{N} \psi\left(\delta_{n}\right) g\left(A_{n}\right)
$$

where $\psi(\delta)$ is the probability density function for the fast cavity phase error and $g(A)$ is the probability density for the voltage error. The probability densities are normalized, meaning that they give probabilities on integration. The function $F$ is used to compute ensemble averages, e. g.

$$
\bar{T}=\int T F\left(E, \Phi, \delta_{1}, A_{1}, \ldots\right) d E d \Phi d \delta_{1} d A_{1} \ldots .
$$

The average energy is

$$
\bar{T}=\bar{E}+\sum_{n=1}^{N} \sum_{i=1}^{k} E_{n} I_{i}\left(\phi_{n}, \Phi_{0}\right)
$$

where

$I_{i}=\int \cos \left(\Phi_{i}+\phi-\Phi_{0}+\Phi+\delta\right) f(E, \Phi) \psi(\delta) d E d \Phi d \delta$

since $\int A g(A) d A=0 . I_{i}$ can be re-expressed as

$I_{i}=\cos \left(\Phi_{i}+\phi-\Phi_{0}\right) I_{c}-\sin \left(\Phi_{i}+\phi-\Phi_{0}\right) I_{s}, i=1, \ldots k$

where

$$
I_{c}=\int \cos (\Phi+\delta) f(E, \Phi) \psi(\delta) d E d \Phi d \delta
$$




\section{DISCLAIMER}

Portions of this document may be illegible in electronic image products. Images are produced from the best available original document. 
and $I_{s}$ is the same integral as above except the cosine function is replaced by the sine function. The vernier sets $\Phi_{0}$ and the cavity excitations so that

$$
\begin{aligned}
\bar{T} & =\bar{E}+E_{f} \sum_{i=1}^{k} \cos \Phi_{i}, \\
\frac{d \bar{T}}{d \Phi_{0}} & =E_{f} \sum_{i=1}^{k} \sin \Phi_{i},
\end{aligned}
$$

i.e. the energy gain of the accelerator is fixed to $E_{f}\left(\cos \Phi_{1}+\ldots+\cos \Phi_{k}\right)$ and the injector phase offset is set so the bunch is at a phase $\Phi_{1}$ during pass $1, \ldots, \Phi_{k}$ during pass $k$, of the accelerator as a whole. This means

$$
\begin{aligned}
& \sum_{n=1}^{N} E_{n}\left[\left(C I_{c}-S I_{s}\right) \cos \left(\phi_{n}-\Phi_{0}\right)-\right. \\
& \left.\left(S I_{c}+C I_{s}\right) \sin \left(\phi_{n}-\Phi_{0}\right)\right]=E_{f} C \\
& \sum_{n=1}^{N} E_{n}\left[\left(S I_{c}+C I_{s}\right) \cos \left(\phi_{n}-\Phi_{0}\right)+\right. \\
& \left.\left(C I_{c}-S I_{s}\right) \sin \left(\phi_{n}-\Phi_{0}\right)\right]=E_{f} S
\end{aligned}
$$

where $C \equiv \sum_{i=1}^{k} \cos \Phi_{i}$ and $S \equiv \sum_{i=1}^{k} \sin \Phi_{i}$. It is now possible to solve for the cavity sums:

$$
\begin{aligned}
& \sum_{n=1}^{N} E_{n} \cos \left(\phi_{n}-\Phi_{0}\right)=\frac{I_{c} E_{f}}{I_{c}^{2}+I_{s}^{2}} \\
& \sum_{n=1}^{N} E_{n} \sin \left(\phi_{n}-\Phi_{0}\right)=-\frac{I_{s} E_{f}}{I_{c}^{2}+I_{s}^{2}}
\end{aligned}
$$

Now,

$$
\overline{T^{2}}=\overline{E^{2}}+2 \bar{E} S_{1}+S_{2}+\left(1+I_{A}\right) S_{3},
$$

where

$$
\begin{aligned}
S_{1}= & \sum_{n=1}^{N} E_{n}\left[C_{n} I_{c}-S_{n} I_{s}\right] \\
S_{2}= & \sum_{n=1}^{N} \sum_{p \neq n}^{N} E_{n} E_{p}\left[C_{n} C_{p} I_{c c}-C_{n} S_{p} I_{c s}-\right. \\
& \left.S_{n} C_{p} I_{s c}+S_{n} S_{p} I_{s s}\right] \\
S_{3}= & \sum_{n=1}^{N} E_{n}^{2}\left[C_{n}^{2} I_{2 c c}+\right. \\
& \left.S_{n}^{2} I_{2 s s}-C_{n} S_{n} I_{2 c s}-S_{n} C_{n} I_{2 s c}\right] ;
\end{aligned}
$$

and

$$
C_{n}=\sum_{i=1}^{k} \cos \left(\Phi_{i}+\phi_{n}-\Phi_{0}\right)
$$

and $S_{n}$ is the same sum as above except the cosine function is replaced by the sine function. $I_{c c}$ is the integral of $\cos (\Phi+\delta) \cos \left(\Phi+\delta^{\prime}\right)$ over the probability densities $f(E, \Phi), \psi(\delta)$ and $\psi\left(\delta^{\prime}\right), I_{c s}$ is the integral of $\cos (\Phi+\delta) \sin \left(\Phi+\delta^{\prime}\right), I_{2 c c}$ and $I_{2 c s}$ are integrals of $\cos (\Phi+\delta) \cos (\Phi+\delta)$ and $\cos (\Phi+\delta) \sin (\Phi+\delta)$ respectively, and $I_{A}=\int A^{2} g(A) d A$. The relative $r m s$ energy spread is given by the sum of four terms

$$
\frac{T_{\mathrm{rms}}}{\bar{T}}=\frac{\sqrt{\bar{T}^{2}}-\bar{T}^{2}}{\bar{T}}=\frac{\sqrt{E_{\mathrm{rms}}^{2}+T_{1}^{2}+T_{2}^{2}+T_{3}^{2}}}{\bar{T}}
$$

where $E_{\mathrm{rms}}$ is the energy spread at injection,

$$
\begin{gathered}
T_{1}^{2}=\sum_{n=1}^{N} \sum_{p=1}^{N} E_{n} E_{p}\left(\lambda_{1}-\lambda_{2}\right) \\
T_{2}^{2}=\sum_{n=1}^{N} E_{n}^{2}\left(\kappa_{1}-\kappa_{2}\right) \\
T_{3}^{2}=I_{A} \sum_{n=1}^{N} E_{n}^{2}\left[C_{n}^{2} I_{2 c c}+S_{n}^{2} I_{2 s s}-C_{n} S_{n}\left(I_{2 c s}+I_{2 s c}\right)\right]
\end{gathered}
$$

where

$$
\begin{aligned}
& \lambda_{1}=C_{n} C_{p}\left(I_{c c}-I_{c}^{2}\right)+S_{n} S_{p}\left(I_{s s}-I_{s}^{2}\right) \\
& \lambda_{2}=C_{n} S_{p}\left(I_{c s}+I_{c} I_{s}\right)+S_{n} C_{p}\left(I_{s c}-I_{s} I_{c}\right) \\
& \kappa_{1}=C_{n}^{2}\left(I_{2 c c}-I_{c c}\right)+S_{n}^{2}\left(I_{2 s s}-I_{s s}\right) \\
& \left.\kappa_{2}=C_{n} S_{n}\left(I_{2 c s}-I_{c s}\right)+S_{n} C_{n}\left(I_{2 s c}-I_{s c}\right)\right]
\end{aligned}
$$

The first term in eq. (9) is from the energy spread at injection and is negligible in the IR DEMO. The second term gives the contributions from the finite bunch length and from the injector phase error. Since these errors are the same at each cavity (correlated errors) the resulting energy spread is independent of the number of cavities. Also the second term is independent of the slow phase errors.

The third term is due to the fast phase errors in the individual cavities and due to the fact that the bunches traverse the individual cavities with the slightly wrong phase because of the slow phase errors. This contribution vanishes if the fast phase errors vanish since in this case $I_{c c}=I_{2 c c}$, etc. For fixed total energy the third term is inversely proportional to the number of cavities. The fourth term in the sum is due to the voltage errors in the system and it is inversely proportional to the number of cavities, the usual result of uncorrelated errors.

In the totally correlated case where we assume that $A_{1}=$ $\ldots .=A_{N}$ and $\delta_{1}=\ldots=\delta_{N}$, the probability density is

$$
\begin{aligned}
F\left(E, \Phi, \delta_{1}, A_{1}, \ldots\right) & =f(E, \Phi) \psi\left(\delta_{1}\right) g\left(A_{1}\right) \\
& \times \prod_{n=2}^{N} \delta\left(\delta_{1}-\delta_{n}\right) \delta\left(A_{1}-A_{n}\right) .
\end{aligned}
$$

The result of calculating $\bar{T}$ is the same as before. However,

$$
\begin{aligned}
T_{1}^{2}= & \frac{E_{f}^{2}}{\left(I_{c}^{2}+I_{s}^{2}\right)^{2}}\left[\left(C I_{c}+S I_{s}\right)^{2}\left(I_{2 c c}-I_{c}^{2}\right)\right. \\
& +\left(S I_{c}-C I_{s}\right)^{2}\left(I_{2 s s}-I_{s}^{2}\right) \\
& \left.-2\left(S I_{c}-C I_{s}\right)\left(C I_{c}+S I_{s}\right)\left(I_{2 c s}-I_{c} I_{s}\right)\right] \\
T_{2}^{2}= & 0
\end{aligned}
$$




$$
\begin{aligned}
T_{3}^{2}= & \frac{I_{A} E_{f}^{2}}{\left(I_{c}^{2}+I_{s}^{2}\right)^{2}}\left[\left(C I_{c}+S I_{s}\right)^{2} I_{2 c c}+\right. \\
& \left.\left(S I_{c}-C I_{s}\right)^{2} I_{2 s s}-2\left(S I_{c}-C I_{s}\right)\left(C I_{c}+S I_{s}\right) I_{2 c s}\right] .
\end{aligned}
$$

Since the $T_{2}$ term is zero, there is no dependency of the result on the slow phase errors $\phi_{n}$. The third term is a factor of $N$ larger than before, as is characteristic in the transition from uncorrelated to correlated errors.

As an example, we assume gaussian distributions of errors and obtain,

$$
\begin{aligned}
I_{c} & =\exp \left(-\sigma_{\Phi}^{2} / 2\right) \exp \left(-\sigma_{i}^{2} / 2\right) \exp \left(-\sigma_{\delta}^{2} / 2\right) \\
I_{c c} & =\left[0.5+0.5 \exp \left(-2 \sigma_{\Phi}^{2}\right) \exp \left(-2 \sigma_{i}^{2}\right)\right] \exp \left(-\sigma_{\delta}^{2}\right) \\
I_{s s} & =\left[0.5-0.5 \exp \left(-2 \sigma_{\Phi}^{2}\right) \exp \left(-2 \sigma_{i}^{2}\right)\right] \exp \left(-\sigma_{\delta}^{2}\right) \\
I_{2 c c} & =0.5+0.5 \exp \left(-2 \sigma_{\Phi}^{2}\right) \exp \left(-2 \sigma_{i}^{2}\right) \exp \left(-2 \sigma_{\delta}^{2}\right) \\
I_{2 s s} & =0.5-0.5 \exp \left(-2 \sigma_{\Phi}^{2}\right) \exp \left(-2 \sigma_{i}^{2}\right) \exp \left(-2 \sigma_{\delta}^{2}\right) \\
I_{c s} & =I_{s c}=I_{s}=I_{2 c s}=I_{2 s c}=0 \\
I_{A} & =\sigma_{A}^{2} .
\end{aligned}
$$

The effects of the finite bunch length and of the injector phase error may be characterized by a single parameter $\sigma_{I}^{2}=\sigma_{\Phi}^{2}+\sigma_{i}^{2}$, an "effective bunch length" which depends on the properties of the injector alone.

We now specify the vernier system in greater detail. As an example assume that all the cavities except one, the $m$-th cavity, are set so

$$
E_{n}=\frac{E_{f} C}{k N} \text { for } n \neq m \text {. }
$$

This means that Eq. (3) becomes

$E_{m} \simeq \frac{E_{f} C}{k N}\left\{1+\frac{N k}{C}-N+\frac{k N}{2 C}\left(\sigma_{I}^{2}+\sigma_{\delta}^{2}\right)+\frac{N \Delta \phi^{2}}{2}\right\}$

and

$$
\Phi_{0} \simeq \sum_{n=1}^{N} \phi_{n} / N
$$

to second order in the small quantities $\sigma_{I}, \ldots$, and $\Phi_{0}$. In the above formula

$$
\Delta \phi^{2}=\sum_{n=1}^{N}\left(\phi_{n}-\Phi_{0}\right)^{2} / N
$$

is approximately the square of the rms average of the slow phase errors since $\Phi_{0}$ is approximately equal to the average of the slow phase errors.

The two estimates give that the relative $r m s$ energy spread satisfies

$$
\sqrt{D_{1}} \leq E_{\mathrm{rms}} / E \leq \sqrt{D_{2}}
$$

where

$$
\begin{aligned}
D_{1} \simeq & \sigma_{I}^{2} \frac{S^{2}}{C^{2}}+\sigma_{\delta}^{2} \frac{S^{2}}{k^{2} N}\left(\frac{2 k}{C}-1\right) \\
& +\sigma_{A}^{2} \frac{C^{2}}{k^{2} N}\left(\frac{2 k}{C}-1\right)+\frac{1}{2} \sigma_{I}^{4}
\end{aligned}
$$

$$
\begin{aligned}
& +\left(\frac{1}{2} \sigma_{\delta}^{4}+\sigma_{I}^{2} \sigma_{\delta}^{2}\right) \frac{C^{2}}{k^{2} N}\left(\frac{2 k}{C}-1\right) \\
& +\frac{\sigma_{\delta}^{2}}{k^{2} N}\left\{\Delta \phi^{2}\left[C^{2}+\frac{S^{2}}{2}+S^{2} N\left(\frac{k}{C}-1\right)\right]\right. \\
& +\left(\sigma_{I}^{2}+\sigma_{\delta}^{2}\right) S^{2}\left(\frac{k}{C}-1\right)\left(\frac{k N}{C}-1\right) \\
& +4 S C\left(\phi_{m}-\Phi_{0}\right)\left(\frac{k}{C}-1\right) \\
& \left.+2\left(\phi_{m}-\Phi_{0}\right)^{2}\left(C^{2}-\frac{S^{2}}{2}\right)\left(\frac{k}{C}-1\right)\right\} \\
& +\sigma_{A}^{2}\left(\sigma_{I}^{2}+\sigma_{\delta}^{2}\right) \frac{S^{2}}{k^{2} N}\left(\frac{2 k}{C}-1\right)
\end{aligned}
$$

and

$D_{2} \simeq\left(\sigma_{I}^{2}+\sigma_{\delta}^{2}\right) \frac{S^{2}}{C^{2}}+\sigma_{A}^{2}+\frac{1}{2}\left(\sigma_{I}^{2}+\sigma_{\delta}^{2}\right)^{2}+\sigma_{A}^{2}\left(\sigma_{I}^{2}+\sigma_{\delta}^{2}\right) \frac{S^{2}}{C^{2}}$,

to fourth order in the small quantities. The two estimates depend on whether the errors are correlated (LH inequality) or uncorrelated (RH inequality).

If we set $S=0$ and $C=k$ for on crest acceleration and $k=1$ for one pass through the accelerator, the expressions above become

$$
\begin{gathered}
D_{1} \simeq \frac{1}{2} \sigma_{I}^{4}+\frac{1}{N} \sigma_{\delta}^{2} \Delta \phi^{2}+\frac{1}{N} \sigma_{\delta}^{2}\left(\frac{1}{2} \sigma_{\delta}^{2}+\sigma_{I}^{2}\right)+\frac{1}{N} \sigma_{A}^{2} \\
D_{2} \simeq \frac{1}{2}\left(\sigma_{I}^{2}+\sigma_{\delta}^{2}\right)^{2}+\sigma_{A}^{2}
\end{gathered}
$$

in agreement with previous results [1].

\section{CEBAF'S IR DEMO: AN EXAMPLE}

Now we calculate the if phase and amplitude tolerances necessary to obtain $4 \times 10^{-4}$ energy spread at the end of the IRFEL linac, where bunches ride $12.5^{\circ}$ off crest and the injection energy is $10 \mathrm{MeV}$ and the final energy is $42 \mathrm{MeV}$. We assume an effective bunch length of $\sigma_{I}=3.96 \times 10^{-4}$. We then split the rest of the energy spread equally between the phase error and amplitude error. We find that the permissible relative voltage fluctuation $\sigma_{A}$ in the correlated case to be $2.8 \times 10^{-4}$ and the permissible fast phase fluctuation $\sigma_{\delta}$ to be $0.07^{\circ}$. The uncorrelated errors are $\sigma_{A} \leq 7.8 \times 10^{-4}$ and $\sigma_{\delta} \leq 0.2^{\circ}$ assuming no vernier, as will be the case in CEBAF's IR DEMO FEL.

\section{ACKNOWLEDGEMENT}

This work was supported by DOE Contract \#DE-AC0584ER40150.

\section{REFERENCES}

[1] G. A. Kraft, CEBAF-TN-0050, May 1987. 